\title{
Systemic inflammation in patients with Takotsubo syndrome: a review of mechanistic and clinical implications
}

\author{
Kenan Yalta ${ }^{1}$, Ertan Yetkin ${ }^{2}$, Tulin Yalta ${ }^{3}$ \\ ${ }^{1}$ Cardiology Department, Trakya University, Edirne; ${ }^{2}$ Cardiology Department, Derindere Hospital, Istanbul; ${ }^{3}$ Pathology \\ Department, Trakya University, Edirne, Turkey
}

\begin{abstract}
Over recent decades, systemic inflammation as quantified with inflammation markers or indices has been extensively investigated in the setting of various cardiovascular conditions including heart failure (HF), acute coronary syndromes (ACS). In contrast, systemic inflammation in patients with takotsubo syndrome (TTS) has been an underrated phenomenon in clinical practice. On the other hand, experimental and clinical data have been rapidly growing in the recent years regarding pathogenetic, prognostic as well as therapeutic implications of systemic inflammation in TTS. Accordingly, the present article aims to provide a general perspective on mechanistic and clinical aspects of systemic inflammation in the setting of TTS.
\end{abstract}

\section{Introduction}

In clinical practice, systemic inflammation is well known to have pathogenetic and prognostic implications in patients with

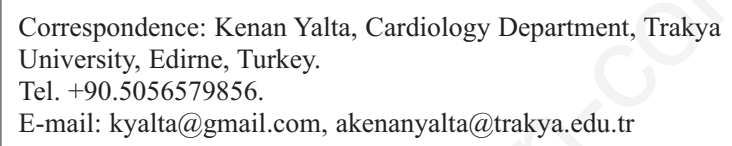

E-mail:kyalta@gmail.com, akenanyalta@trakya.edu.tr

Key words: Takotsubo syndrome, systemic inflammation, inflammation markers, inflammation indices, mechanisms, clinical implications. Contributions:

Authors contribution: KY, EY, TY, design, conception, acquisition of literature data, analysis, interpretation, substantial writing, drafting, final revision and approval, affirming responsibility for the final content.

Conflict of interest: The authors declare that they have no competing interests, and all authors confirm accuracy.

Received for publication: 4 December 2020.

Accepted for publication: 10 January 2021.

${ }^{\circ}$ Copyright: the Author(s), 2021

Licensee PAGEPress, Italy

Monaldi Archives for Chest Disease 2021; 91:1718

doi: 10.4081/monaldi.2021.1718

This article is distributed under the terms of the Creative Commons Attribution Noncommercial License (by-nc 4.0) which permits any noncommercial use, distribution, and reproduction in any medium, provided the original author(s) and source are credited. cardiovascular conditions including acute coronary syndromes (ACS), heart failure (HF) as well as atrial fibrillation (AF) [1]. Based on recent data, this might also apply to the setting of takotsubo syndrome (TTS) that is considered as a transient form of myocardial dysfunction primarily associated with a variety of emotional and physical triggers [2-4]. As analogous to ACSs, TTS mostly presents with a variety of symptoms including chest pain, dyspnea, etc. and certain electrocardiographic (ECG) findings including precordial ST segment elevation, T-wave inversion [2]. Characteristically, myocardial dysfunction in patients with TTS extends beyond the territory of a single coronary artery usually in a circumferential manner (mostly in the absence of a culprit coronary stenosis), and manifests itself as a left ventricular (LV) apical ballooning pattern (classical form), and occasionally in the form of atypical variants (namely midventricular, reverse, focal and diffuse variants) [2]. Mechanistically, sudden myocardial stunning usually in response to adrenergic discharge (characterized by moderate levels of myocardial enzymes, significant levels of ventricular stretch markers including $\mathrm{N}$ terminal pro-brain natriuretic peptide (NT-proBNP) and absence of permanent myocyte death) possibly accounts for these characteristic wall motion abnormalities in these patients [2]. However, pathogenesis of this phenomenon is likely multifaceted, and has yet remained to be fully established [2-4]. More importantly, the disease course may not be so lenient (as opposed to previous considerations) leading to a variety of in-hospital complications including malignant arrhythmogenesis and mechanical complications [2].

Besides the rapidly growing information on risk-stratification and management of patients with TTS [2], there might also exist particular implications of systemic inflammation in this specific population. Mechanistically, systemic inflammation in the setting of TTS might be attributable to multiple sources or triggers. More importantly, it seems quite likely that, on top of well-known risk stratifiers including advanced age, low left ventricular ejection fraction (LVEF), an existing physical stressor, etc. [2], evaluation of inflammation markers and indices might enable further riskstratification and hence; proper management of TTS [3]. In this review article, we aim to highlight mechanistic and clinical implications of systemic inflammation in patients with TTS.

\section{Systemic inflammation in TTS: Primary sources and triggers}

In this context, systemic inflammation possibly has certain temporal and mechanistic characteristics. Accordingly, sytemic inflammation in patients with TTS might be due to a variety of pre-existing and/or new-onset factors including adrenergic dis- 
charge, pre-existing inflammatory stressors, myocardial inflammation, enhanced myocardial wall stress (due to myocardial dysfunction, mechanical complications including acute left ventricular outflow tract (LVOT) gradient), and occasionally; co-existing acute cardiac conditions including ACSs and myocarditis [1-9].

\section{Severe adrenergic discharge}

Adrenergic discharge, as is widely considered, appears to be the hallmark of TTS evolution [2]. Furthermore, adrenergic discharge might persist far beyond the onset of this phenomenon particularly in cases complicated by acute HF or mechanical complications [2]. Importantly, severe adrenergic discharge (as determined with excessive levels of serum catecholamines and presence of specific findings including coronary slow flow (CSF) phenomenon (in major epicardial coronary arteries), measured using TIMI frame counts (TFCs) on invasive coronary angiogram (CAG) $[2,4]$ might be regarded as the main trigger of systemic inflammation in certain patients with TTS, particularly in the absence of an overt inflammatory source.

Mechanistically, catecholamines might potentiate systemic inflammatory response particularly in the setting of infections (including sepsis) and tissue injury [10-12]. Impact of adrenergic system on systemic inflammation is largely mediated through adrenoreceptors expressed on a variety of inflammatory cells including neutrophils, monocytes, macrophages as well as lymphocytes $[10,13]$. In particular, substantial levels of adrenaline were previously demonstrated to induce the release of certain pro-inflammatory cytokines including interleukin-6 (IL-6), IL-1 $\beta$ and tumor necrosis factor- $\alpha$ (TNF- $\alpha)$ through stimulation of both alpha $(\alpha)(\alpha-$ 1 and 2 subtypes) and beta ( $\beta$ ) ( $\beta-1,2$ and 3 subtypes) adrenoreceptors $[10]$. In particular, severe adrenergic discharge was previously reported to be associated with the emergence of pro-inflammatory macrophage phenotype due to induction of $\beta-2$ adrenoreceptors leading to oversecretion of IL-6 and exaggerated neutrophil trafficking in an experimental model [10]. Therefore, blockade of $\beta-2$ adrenoreceptor signaling and/or IL-6 might have important therapeutic implications in the management of systemic inflammation, at least, primarily driven by pro-inflammatory macrophages [10]. Likewise, substantial levels of cytokines, including IL-6, were previously reported in the setting of physically and emotionally stressful scenarios characterized by adrenergic hyperactivation including pscyhological stress, burn injuries [10] that all might be associated with TTS evolution in the clinical setting.

Interestingly, certain reports suggest opposite effects of adrenoreceptor subtypes on cytokine production under physiological conditions $[13,14]$. Accordingly, $\beta$ adrenoreceptors (mostly $\beta$ 2 subtype), as opposed to $\alpha$ adrenoreceptors, might have a significant inhibitory impact on the production of certain pro-inflammatory cytokines including TNF- $\alpha$ along with their potential to induce the production of anti-inflammatory cytokines including IL-10 in healthy subjects $[13,14]$. Therefore, diverse characteristics of adrenoreceptors in the setting of systemic inflammation might ensure stable cytokine levels in healthy subjects even when they are exposed to short-term adrenergic discharge.

On the other hand, a significant rise in pro-inflammatory cytokine levels is generally encountered in the setting of HF possibly attributable, in part, to the generalized $\beta$ adrenoreceptor downregulation with consequent unopposed impact of $\alpha$ adrenoreceptors on pro-inflammatory cytokine production $[13,14]$. Accordingly, noradrenaline and isoproterenol-induced inhibition of TNF- $\alpha$ production - by the lipopolysaccharide (LPS)-stimulated monocytic THP-1 cells and whole blood cultures - was previously demonstrated to be significantly reduced in patients with HF compared with healthy controls $[13,14]$. Therefore, generalized alterations in the density of adrenoreceptor subtypes might also contribute to the pro-inflammatory status in patients with HF.

More specifically, a recent immunohistochemical study has demonstrated, for the first time, significant $\beta$ adrenoreceptor downregulation (as determined with overexpression of $G$ proteincoupled receptor kinase 2 (GRK2) and $\beta$-arrestin- 2$)$ in patients with TTS as part of protective mechanisms in response to excessive catecholamine levels [15]. Interestingly, the degree and extent of $\beta$ adrenoreceptor downregulation was more significant in these patients compared with patients with dilated cardiomyopathy (DCMP) [15]. Therefore, $\beta$ adrenoreceptor downregulation in patients with TTS might potentially lead to overproduction of proinflammatory cytokines due to unopposed $\alpha$ adrenoreceptor actions on inflammatory cells. However, the issue of whether $\beta$ adrenoreceptor downregulation in the setting of TTS emerges as a local (confined to myocardium) or generalized phenomenon remains to be established.

On the other hand, impact of adrenergic discharge on systemic inflammation might also be attributable to changes in blood and lymph flow along with altered distribution and production of proinflammatory cells [16]. In this context, sympathetic innervation of immune organs (bone marrow, spleen, timus, etc.) [17] possibly accounts for the over-production of pro-inflammatory cells along with their consequent spillover into the circulation during adrenergic hyperactivation (as in the setting of TTS).

Conversely, circulating cytokines might also have the potential to stimulate adrenergic system and hypothalamo-pituitary-axis (HPA) largely through CNS signaling as part of stress management strategies including energy production (through utilization of fatty acids, glucose) for the increased demands of activated pro-inflammatory cells [16]. Therefore, there seems to be a positive loop between adrenergic discharge and systemic inflammation, at least, at the systemic level. Taken together, the above-mentioned notions might potentially suggest adrenergic discharge as a strong trigger of systemic inflammation in patients with TTS. Nevertheless, potential association between adrenergic system and systemic inflammation has been investigated mostly in experimental models, and requires further clinical research.

\section{Pre-existing inflammatory stressors}

A variety of pre-existing inflammatory conditions including infections, malignancy, major surgery, major burns (serving as TTS triggers or by-stander conditions) might also arise as important sources of systemic inflammation potentially exhibiting a close correlation with adrenergic discharge in the setting of TTS $[2,4]$. In a previous report, 27 out of 114 patients with TTS were diagnosed as having cancer at the time of TTS diagnosis or on follow-up [7]. The authors suggested a potentially common origin of these two conditions [7]. However, TTS patients with cancer had higher levels of C-reactive protein (CRP) along with higher prevalence of inflammation-related arrhythmias including AF in this study [7] suggesting malignancy as an important trigger of systemic inflammation, and in particular, a strong physical stressor for TTS evolution largely through enhancement of myocardial adrenoreceptor sensitivity $[6,18]$. Similarly, cancer prevalence in another study was found to be significantly higher in the setting of TTS as compared with acute 
myocardial infarction (AMI) suggesting the strong association of paraneoplastic factors with TTS evolution [18]. In other terms, severe systemic inflammation in the setting of cancer might have the potential to induce spontaneous TTS episodes irrespective of apparent stressors (emotional, etc.) [6].

Similarly, severe infections including sepsis might induce TTS due to adverse effects of cytokines (and, to some extent, prostaglandins and free radicals) generally manifesting as myocardial contractile dysfunction and adrenoreceptor hypersensitivity [6,18-21]. In contrast, septic cardiomyopathy (S-CMP) (a more common phenomenon generally presenting with a global and reversible myocardial dysfunction in septic patients) [22] was previously suggested to be characterized by myocardial adrenergic hyporesponsiveness, reduced sensitivity of myofilaments to calcium, mitochondrial dysfunction and excessive nitric oxide (NO) levels (mostly through induction of inducible nitric oxide synthase (NOS) (NOS-2) in response to endotoxins) $[19,23]$.

Based on the above-mentioned notions, it might be suggested that pattern of myocardial adrenoreceptor responsiveness (hypo or hyper), possibly influenced by individual variations in synthesis and release of key mediators, might also determine the clinical presentation pattern of acute myocardial dysfunction (TTS or SCMP) in patients with sepsis. Within this context, besides the well known impact of excessive NO levels on beta adrenergic hyporesponsiveness (possibly associated with alterations in sarcolemmal calcium channels [19]), PG-E2 might also impair myocardial contractility in response to adrenergic stimulation through inhibition of phosphodiesterase-4 [23]. Taken together, excessive levels of certain mediators (including NO) in the setting of sepsis might be associated with the evolution of S-CMP through induction of myocardial $\beta$ adrenoreceptor hyporesponsiveness. On the other hand, TTS in patients with sepsis is characterized by $\beta$ adrenoreceptor hypersensitivity, and possibly presents with moderate levels of specific mediators including NO. More detailed information on mechanisms of myocardial dysfunction in the setting of critical diseases may be found elsewhere [19,23].

Other less common, yet; serious clinical conditions including anaphylaxis (characterized by extreme vascular permeability in response to certain allergens, and relatively different type of mediators), Kounis syndrome (allergic coronary vasospasm and/or AMI) and hemophagocytic lymphohistiocytosis (HLH) (characterized by lysis of blood cells by the activated self macrophages and lymphocytes leading to bouts of cytokine storm associated with multiorgan dysfunction) might occasionally serve as inflammatory physical stessors in certain cases with TTS [20]. Cytokine-mediated mechanisms of TTS evolution might also apply to the setting of other specific conditions including the latest pandemic coronavirus disease 2019 (COVID-19) particularly in the presence of severe clinical presentations including cytokine release syndrome (CRS) [24]. In this context, CRS has been usually regarded as a late-onset phenomenon mostly in fragile subjects who have impaired cellular immune mechanisms to combat the pathogen at the relatively earlier stages of COVID-19 [25]. An updated report of TTS cases in patients with COVID-19 has been recently published [26]. Therefore, TTS should be kept in mind as a relatively rare, yet; possible cause of acute myocardial dysfunction in patients with COVID-19, and might be associated with a variety of physical (including virus-related hyperinflammation) and emotional (including fear of dying, social isolation) stressors in these patients [26].

Occasionally, certain by-stander inflammatory conditions (chronic rheumatological or autoimmune diseases, in-hospital infectious complications (following a TTS admission) that are not considered to account for the evolution of a particular TTS episode) might significantly contribute to systemic inflammatory response in patients with TTS. However, a pre-existing inflammatory condition might not serve as an entirely by-stander phenomenon even in the presence of other overt TTS stressors. Accordingly, a background chronic inflammatory state was previously suggested to lower the threshold for the evolution of TTS in response to emotional and physical triggers [4].

Taken together, pre-existing inflammatory conditions in the setting of TTS mostly serve as physical stressors associated with variable degrees of systemic inflammation and adrenergic discharge. As described previously, systemic inflammatory response also emerges in the setting of various emotional conditions [10]. In other terms, systemic inflammation and adrenergic system, regardless of the type of the trigger, work harmoniously in the evolution of TTS. However, as described later, pre-existing inflammatory states should not simply be regarded as triggers of TTS, but also as conditions with prognostic impact both in the short and long term.

\section{Myocardial inflammation: A novel concept with important implications}

Importantly, myocardial inflammation in patients with TTS has risen as a novel entity, and might also serve as an important source of systemic inflammation in these patients [8,9,20,27-29]. Within this context, myocardial edema throughout the whole RV myocardium (possibly due to myocardial inflammation) on magnetic resonance imaging (MRI) was previously reported to persist at 4 months in a population of 31 TTS patients with ST segment elevation on ECG [28]. Similarly, reduction in the values of global longitudinal strain (GLS) (an echocardiographic marker of subclinical myocardial dysfunction) were demonstrated to persist at 3 months in a population of 36 female patients with TTS potentially suggesting the impact of residual myocardial inflammation [29]. Mechanistically, nitrosative stress (triggered by certain factors including endotoxins and adrenergic discharge) as determined with increased NO bioavailability (due to overexpression of NOS) along with enhanced NO responsivenes might account for myocardial vascular permeability leading to plasma leakage and infiltration of specific pro-inflammatory cells in the interstitial space $[20,23]$. Certain peptides associated with adrenergic system might also contribute to local myocardial inflammation during adrenergic hyperactivation. Accordingly, neuropeptide-Y (co-released with noradrenaline from sympathetic nerve endings) was previously shown to enhance adhesion of inflammatory cells to endothelium [17] potentially facilitating recruitment of inflammatory cells in the extravascular space. More interestingly, natriuretic peptides (including BNP) were previously suggested to inhibit the formation of reactive oxygen species (through modulation of neutrophil burst) along with significant suppression of nitrosative stress [20]. Accordingly, considerable BNP levels might not only arise as a consequence of ventricular overstretch but also as a counterbalancing and protective factor against myocardial inflammation in the setting of TTS [20]. Therefore, elevation of natriuretic peptide levels might be regarded as an indirect evidence of myocardial inflammation in patients with TTS [20].

Histopathologically, myocardial biopsy findings in cases with TTS have revealed particular infiltration of neutrophils and macrophages along with expansion of interstitial space, contraction band necrosis as well as findings indicative of significantly diminished energy production (namely metabolic shut-down) including 
increased glycogen content and mitochondrial shape distortion in cardiac myocytes $[2,20]$. Despite rapid recovery of systolic functions, myocardial inflammation was previously reported to persist up to 3 months in TTS cases [20]. Importantly, these histopathological findings were accompanied by activation of survival and antiapoptotic pathways (including overexpression of phosphoinositide3 kinases $(\mathrm{PI} 3 \mathrm{~K})$ ) that provide protection against myocyte death [20]. Experimental models of TTS have also demonstrated significant infiltration of pro-inflammatory macropahages (M1 type), but not anti-inflammatory and reparatory macrophages (M2 type), that primarily account for the release of pro-inflammatory cytokines and degradation of intercellular matrix [20,27]. In a recent experimental rat model, early infiltration of neutrophils was soon replaced by the predominance of M1 macrophages (within days) without a late switch to M2 macrophages (a characteristic finding in the setting of AMI potentially accounting for tissue healing and myocardial remodeling) [27]. Interestingly, these findings were accompanied by an insignificant rise in circulating cytokine levels [27]. Importantly, the authors of this study also reported similar histopathological findings in myocardial specimens of two postmortem TTS hearts [27].

More strikingly, the above-mentioned observations have been recently confirmed by a landmark clinical study that has demonstrated local myocardial inflammation characterized by significant infiltration of M1 type macrophages (as demonstrated with multiparametric cardiac MRI using particles of iron oxide -USPIO) probably as a consequence of adrenergic discharge in patients with TTS [8]. Interestingly, persistent systemic inflammatory response as determined with increased pro-inflammatory monocyte $\left(\mathrm{CD} 14^{++} \mathrm{CD} 16^{-}\right)$count and serum cytokine levels (including CXCL1 (chemokine ligand-1), IL-8 and IL-6) was also demonstrated to persist at 5 months in these patients [8]. However, local myocardial inflammation, though potentially characterized by a protracted course, was suggested as an ultimately reversible phenomenon in this study [8] and in other previous MRI studies investigating myocardial characteristics of TTS based on various parameters of myocardial inflammation [30-32]. Accordingly, lowgrade systemic inflammation might persist even after complete recovery of myocardial contractility, and might be associated with chronic exercise intolerance $[8,9,20]$ potentially attributable to inflammation- mediated subclinical myocardial dysfunction frequently encountered among survivors of TTS [2,29]. Borderline elevation in natriuretic peptide levels within the first few months following TTS recovery might also suggest ongoing myocardial inflammation [20] and associated subclinical myocardial dysfunction in these patients. Accordingly, these findings might provide important therapeutic implications (including antiinflammatory strategies) both in the acute and post-recovery stages of TTS $[8,20,27]$. Taken together, it seems possible that myocardial inflammation might directly contribute to the evolution of characteristic wall motion abnormalities in patients with TTS, and might have a potential association with persistent subclinical myocardial dysfunction in these patients.

Finally, myocardial inflammation might also exert its effects through indirect mechanisms in TTS evolution: It is noteworthy that local inflammation was previously demonstrated to stimulate CNS signaling with consequent adrenergic discharge mostly through cytokine-mediated nociceptor (defined as a receptor activated by local noxious triggers including pain, stretch, etc.) signaling and activation of afferent vagal nerves [16]. Cardiac nociceptor signaling (through receptors including transient receptor potential vanilloid-1 (TRPV1)) has been a well known phenomenon in the setting of various conditions including myocardial ischemia and pericarditis, and is transmitted through afferent spinal and vagal fibers activated by a wide array of mediators including histamine, capsaicin, adenosine and prostaglandines. Importantly, spinal afferent nerves innervating the anterior territory of the LV is strongly associated with reflex adrenergic discharge [30-33]. Since pattern of LV involvement in the setting of TTS is mostly circumferential generally affecting the anterior territories, local myocardial inflammation in this setting might have the potential to induce severe reflex adrenergic discharge through nociceptor signaling, as analogous to anterior AMI [33], along with enhanced adrenoreceptor hypersensitivity through paracrine impact of cytokines.

In summary, myocardial inflammation has important histopathological implications along with its potential pathogenetic role in the setting of TTS through direct and indirect mechanisms (myocardial edema, cytokine impact and nociceptor signaling). Of note, the presence, degree as well as the extent of myocardial inflammation possibly exhibits an individual variation among patients with TTS. In this context, even though recent MRI findings strongly suggest an existing myocardial inflammation [8], previous nuclear imaging studies using specific radiotracers including Gallium-67 $\left({ }^{67} \mathrm{Ga}\right)$ found no evidence of myocardial inflammation in certain TTS cases [34,35]. More importantly, the issue of whether myocardial inflammation serves as a strong contributor or just as an 'epiphenomenon' in TTS evolution still remains to be fully established [20]. In other terms, it seems quite hard to draw firm conclusions regarding absolute contribution of myocardial inflammation to systolic dysfunction in the affected myocardial territories. Traditionally, complete metabolic shut-down due to sudden adrenergic stunning (as confirmed with histopathological and nuclear imaging findings) is generally held responsible for characteristic wall motion abnormalities in TTS (mostly presenting with segmentary akinesis) $[2,20,35]$. In this classical scenario, myocardial inflammation seems to add little or none to the already existing severe myocardial stunning. However, myocardial inflammation might further aggravate myocardial dysfunction in those with partial metabolic shut-down at the very onset of TTS. More importantly, since significant recovery of myocardial inflammation might be much slower as compared with restoration of myocellular metabolic functions [20], TTS patients with a significant degree of myocardial inflammation might possibly have a more protracted disease course characterized by delayed normalization of wall motion abnormalities. As described earlier, subclinical myocardial dysfunction (possibly involving both ventricles) might also persist up to several months in these patients after complete recovery of overt systolic dysfunction $[8,9,20)$.

Interestingly, myocardial inflammation in patients with TTS might also affect apparently normal myocardial segments [20] in a more subtle manner. Moreover, myocardial inflammation in the unaffected segments migh be more severe in the presence of preexisting inflammatory stressors (sepsis, etc.), and might potentially impair compensatory hyperkinesis (that is of crucial importance in the early disease course) in these segments potentially further worsening global systolic functions. However, the above-mentioned notions should be tested through further imaging studies using sophisticated tools including nuclear imaging modalities (using specific radiotracers of local inflammation and cellular metabolism including ${ }^{67} \mathrm{Ga}$, Fluorine-18-labeled fluorodeoxyglucose ( $\left.{ }^{18} \mathrm{~F}-\mathrm{FDG}\right)$, etc. [35].

Figure 1 demonstrates a simplifed illustration of possible interaction among systemic inflammation, myocardial inflammation and adrenergic system in the evolution of TTS. 


\section{Enhanced myocardial wall stress}

It seems quite likely that enhanced wall stress both in the affected and apparently healthy myocardial segments might also serve as a potential contributor to systemic inflammation in patients with TTS. In a previous multicentre study comprising 107 patients with TTS, enhanced myocardial wall stress was suggested to be associated with moderately elevated brain natriuretic peptide (BNP) levels (a biomarker reflecting myocardial wall tension) that might also correlate with inflammation markers [36]. In the general context of HF (and particularly LV pressure overload), myocardial expression and release of certain cytokines (including IL-18 and TNF- $\alpha$ ) and growth factors are well known to be augmented as part of pro-inflammatory signaling potentially contributing to the emergence and perpetuation of HF largely through induction of adverse myocardial remodeling [37,38]. This type of mechanically-induced cytokine release possibly applies to the setting of TTS that was previously reported to have a susbstantial myocardial wall stress in the apical as well as other segments of the $\operatorname{LV}[2,36]$.

Of note, the above-mentioned mechanisms might emerge in a more striking manner in patients with TTS attributable to a pure mechanical basis (mostly arising due to sudden increases in midventricular gradient leading to an apical ballooning pattern $[2,39,40])$ or in those incurring certain mechanical complications including acute LVOT gradient (leading to an abrupt pressure overload in the LV usually without myocardial preconditioning to such pressure elevations).

\section{Co-existing cardiac conditions}

Even though the initial definition of TTS was based on the absence of critical coronary stenosis suggestive of ACSs [2], a por- tion of patients with TTS might suffer co-existing ACSs on admission or during the hospital stay in clinical practice [2,41]. A variety of risk factors (including presence of spontaneous coronary artery dissection (SCAD) on CAG, severe systemic inflammation, presence of physical stressors, intractable chest pain and frailty) have been previously suggested for this particular co-existence [41]. Pathogenetically, TTS and ACS might originate from a common trigger (for instance; an existing severe physical stressor), or one might account for the evolution of the other [41]. Given the well-known impact of ACSs on systemic inflammation [1], a particular TTS episode co-existing with any form of ACSs might be associated with more pronounced systemic inflammation as compared with TTS in isolation.

Similarly, myocarditis (or myopericarditis) might arise as another co-existing phenomenon that might serve as an important source of systemic inflammation in patients with TTS suggesting important diagnostic and prognostic implications as well [42]. In this co-existence, TTS as the primary pathology might occasionally extend pericardium leading to an inflammatory pericardial reaction (myopericarditis) [43]. More rarely, TTS might trigger a process of chemical myocarditis due to excessive levels of circulating catecholamines that might potentially lead to persistent or permanent myocardial damage [42]. In other terms, underlying extreme adrenergic surge might have the potential to induce both TTS and catecholamine-mediated chemical myocarditis in rare instances. In the clinical setting, extreme adrenergic surge is well known to be associated with life-threatening organic causes including subarachnoid hemorrhage and pheochromocytoma. A case of pheochromocytoma- induced myocarditis was previously reported in a young male presenting with reversible diffuse myocardial edema and LV hypertrophy along with persistent midwall late gadolinium enhancement (LGE) [44]. Furthermore, organic causes of adrenergic surge might also trigger TTS evolution, and are no more regarded as exclusion criteria for TTS diagnosis potentially suggesting co-existence of TTS and chemical myocarditis in certain settings [2].

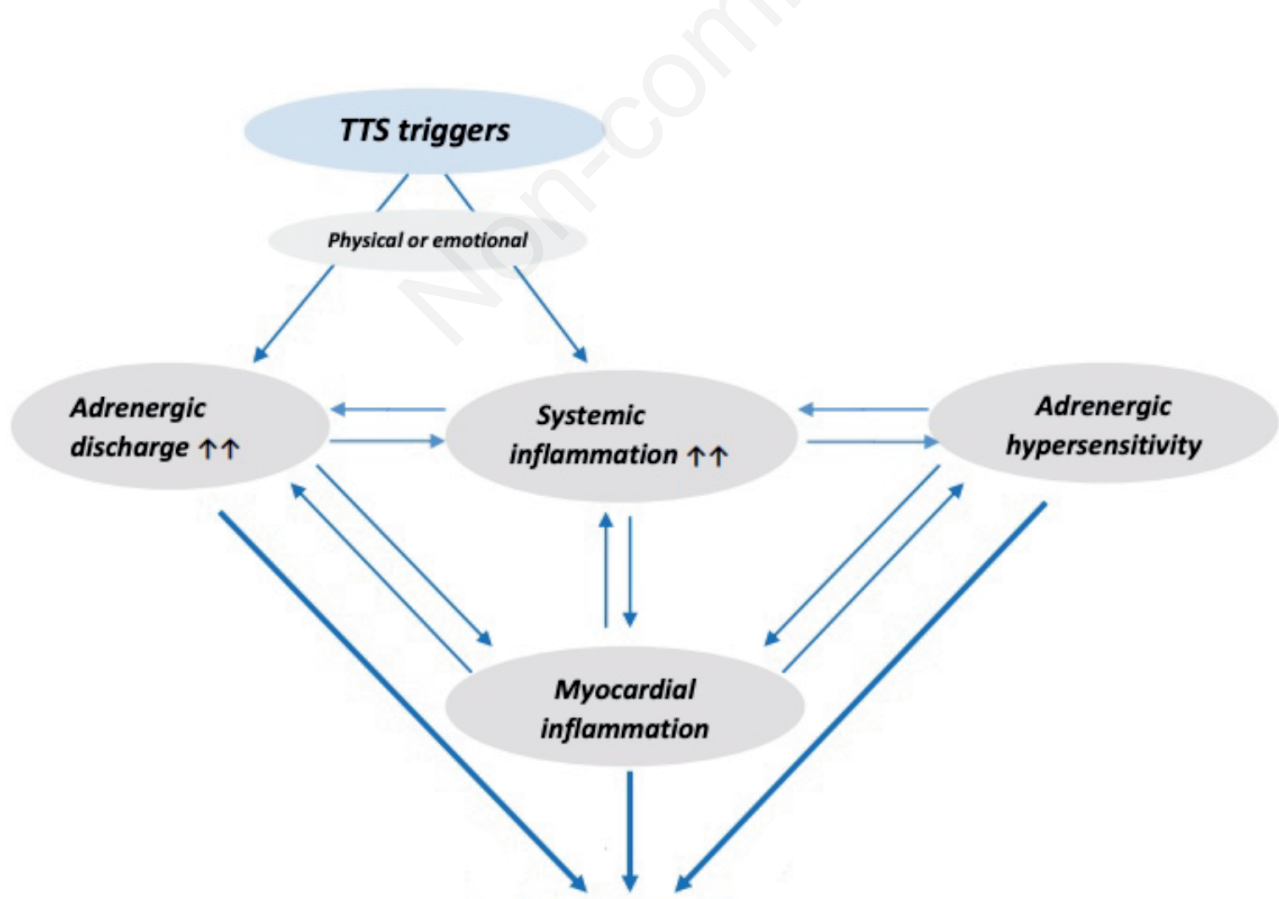

TTS evolution

Figure 1. Potential interaction among systemic inflammation, myocardial inflammation and adrenergic system in TTS (takotsubo syndrome) evolution. 
More often, viral myopericarditis as the primary pathology might also elicit a TTS episode due to certain stressors including intractable chest pain, hemodynamic compromise [42]. On the other hand, co-existing cardiac conditions might possibly go unnoticed in patients with TTS potentially warranting a high index of suspicion along with the use of sophisticated tools including MRI (with LGE assessment) for diagnosis [41,42]. We also strongly encourage the use of MRI particularly in the setting of a documented organic cause or signs of severe adrenergic discharge (presence of severe CSF, extreme LVOT gradient or very high levels of serum catecholamines) to uncover a possible co-existing myocarditis in patients with TTS.

Primary sources and triggers of systemic inflammmation in the setting of TTS are demonstrated in Figure 2. Importantly, there might exist multiple sources of systemic inflammation mostly serving as contributors to and/or consequences of disease process in the setting of TTS evolution. As described in the next section, excessive systemic inflammation might also have prognostic implications beyond its pathogenetic role in patients with TTS.

\section{Inflammation markers and indices: Prognostic implications in TTS}

Systemic inflammation might be associated with important prognostic implications in patients with TTS particularly in those with substantial levels of inflammation markers: In other terms, the severity of systemic inflammation as quantified with serum levels of certain markers or indices (white blood cell (WBC) count, neutrophil/lymphocyte ratio (NLR) as well as mean platelet volume (MPV) as an indirect marker of systemic inflammation) might predict adverse clinical outcomes in these patients [3]. Accordingly, WBC count and BNP were previously suggested as independent predictors of in-hospital adverse events (primarily defined as death, pump failure and malignant arrhythmias including ventricular tachycardia) in patients with TTS [36). Moreover, a recent study has documented independent associations of NLR and ratio of WBC/MPV on admission with in-hospital adverse clinical events (mortality, stroke, cardiogenic shock, etc.) in patients with TTS [3]. In this study, a single NLR value of $>5$ on admission was suggested to predict adverse events in these patients with a sensitivity of $82 \%$ and a specificity of $54 \%$ (with negative and positive predictive values of $84 \%$ and 54\%, respectively) [3]. This may suggest that a single NLR value might primarily serve as a rule-out rather than a rule-in marker of adverse prognosis in the setting of TTS [3] suggesting the need for combined evaluation of inflammation indices or markers to improve positive predictive value of systemic inflammation in this setting.

In another recent study, serum levels of IL-6 and IL-10 on admission were suggested to be significantly associated with adverse prognosis on follow-up in patients with TTS [5]. Of note, elevation of both markers elicited a significantly increased risk for

\section{TAKOTSUBO SYNDROME}

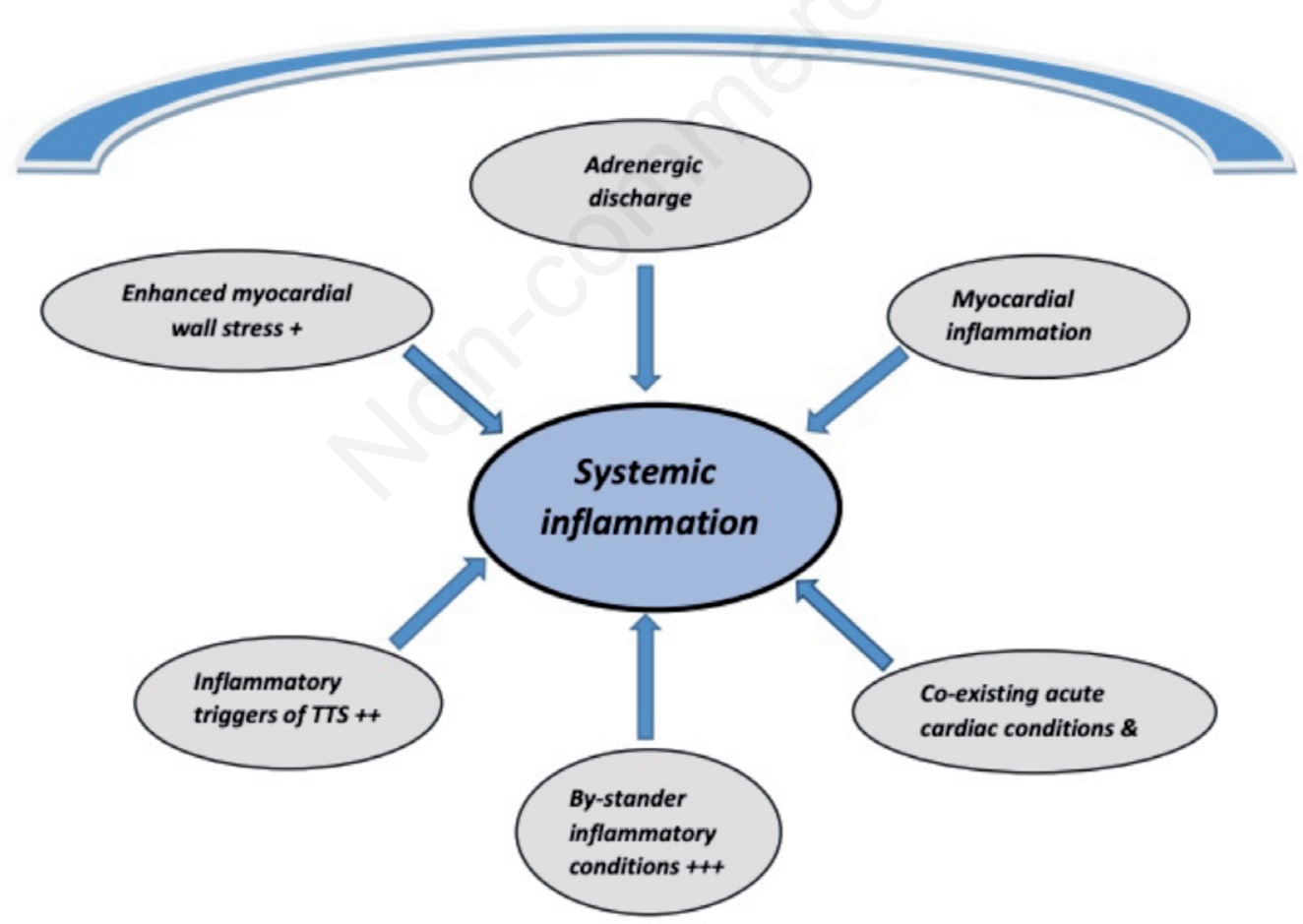

+, Due to severe myocardial dysfunction, mechanical complications including outflow tract gradient, etc.

+ , Including severe infections and malignancy.

,++ Pre-existing (rheumatological diseases,etc.) or new-onset i(n-hospital infectious complications, etc) conditions that are not primarily associated with the evolution of the TTS episode.

Figure 2. Primary sources or triggers of systemic inflammation in patients with \&, Including acute myocardial infarction and myocarditis takotsubo syndrome (TTS). 
adverse events as compared with sole elevation of IL-6 or IL-10 [5] potentially highlighting the value of multi-marker strategy for proper risk-stratification of TTS. It seems likely that duration of pre-existing systemic inflammatory states (including cancer [7] and rheumatological diseases) might also be independently associated with the occurence and severity of adverse events in the acute stage of TTS. However, current information regarding prognostic value of systemic inflammation in patients with TTS still needs to be substantiated through further research.

\section{Mechanisms of inflammation-related adverse events in patients with TTS}

Systemic inflammation might exert its adverse effects through direct and indirect mechanisms in the setting of TTS as analogous to the setting of other cardiovascular conditions:

\section{Direct impact of cytokines}

It is well known that cytokines including IL- $1 \beta$, TNF- $\alpha$ and CRP might exert direct arrhythmogenic effects through abnormal myocardial calcium handling (increased intracellular calcium levels leading to increments in action potential (APD) duration), sodium channel dysfunction (associated with impaired myocardial depolarization), reduction in heart rate variability (HRV), QT interval prolongation and disturbance of ventricular repolarization parameters [1,45-48]. Moreover, endotoxemia associated with gram negative sepsis was previously shown to induce various arrhythmias in experimental models due to its direct impact on Ltype calcium channels along with its potential effects on toll-like receptor-4 located on monocytes leading to excessive cytokine release $[1,49,50)$. Therefore, these arrhythmogenic mechanisms might be quite likely in the setting of TTS associated with sepsis. In general, these findings, to some extent, susbstantiate the higher incidence of malignant arrhythmogenesis (ventricular tachycardia, etc.) previously reported in TTS cases with severe systemic inflammation [3,29].

Serious clinical scenarios including acute respiratory distress syndrome (ARDS) might also arise in a portion of patients with TTS largely due to destructive effects of pro-inflammatory cytokines [51,52]. ARDS is well known to be associated with substantial vascular permeability and tissue damage in various organ systems including pulmonary bed, kidneys, subcutaneous tissue, etc., leading to multi-organ failure in a substantial portion of cases. In TTS patients with ARDS, systemic inflammation generally arises as the primary pathology associated with severe infections, and hence; serves as the common trigger of ARDS and TTS. In other terms, TTS in the setting of ARDS might be regarded just as an 'epiphenomenon' generally with little impact on the final outcome.

\section{Indirect mechanisms}

Indirect mechanisms of systemic inflammation might play a more pivotal role in the evolution of adverse events: within this context, systemic inflammation are well known to enhance coagulation cascade along with supression of fibrinolytic system $[1,53]$ potentially suggesting increased risk for apical thrombus formation and embolic events in patients with TTS with severe systemic inflammation [3]. In particular, certain mediators including Factor$\mathrm{V}$ and complement-3 (C-3) were previously reported to increase in TTS cases compared with controls [54]. Importantly, activation of coagulation and systemic inflammation pathways was also found to be comparable between TTS and AMI groups (potentially suggesting similar rates of adverse events) in this study [54]. Interestingly, coagulation markers were previously suggested to be associated with cardiac arrhythmias in a variety of cardiovascular disorders including AMI and cardiomyopathies [1], and hence; might also serve as indirect arrhythmogenic triggers in TTS patients with a significant inflammatory burden. However, arrhythmogenic impact of these markers still remains to be established in patients with TTS.

Importantly, ACS might also arise in the setting of severe inflammation as a consequence of atherosclerotic plaque rupture, activation of coagulation cascade [1] and coronary microvascular dysfunction, and might potentially aggravate the prognosis in a portion of patients with TTS through potential aggravation of existing HF and arrhythmias. As described earlier, ACS and TTS should not be regarded as mutually exclusive phenomena, and might potentially co-exist in a portion of cases particularly in those with aforementioned risk factors [41,55].

On the other hand, CSF pattern on invasive CAG was previously suggested as an important prognostic marker, and might be attributable to acute coronary microvascular dysfunction associated with adrenergic discharge and systemic inflammation in the setting of TTS $[4,56,57]$. Consistent with this, adverse effects of systemic inflammation in patients with TTS might, to some extent, be dependent on the evolution of CSF phenomenon and consequent coronary ischemic complications $[4,56]$. Therefore, the presence, extent and severity of CSF pattern should be carefully evaluated in patients with TTS, particularly in those with severe systemic inflammation $[4,56]$. Moreover, since the severity of systemic inflammation might generally correlate with the degree of underlying adrenergic discharge $[3,10]$, TTS patients with substantial systemic inflammation might also incur life-threatening consequences of severe adrenergic discharge including catecholaminemediated arrhythmogenesis and acute LVOT gradient $[19,58,59]$.

Undoubtedly, the type, location as well as the extent of underlying inflammatory stressors (malignancy, infections) associated with TTS might directly impact the overall prognosis both in the short and long term possibly in correlation with the severity of associated systemic inflammation. For instance; the more severe the systemic inflammation in a TTS episode primarily attributable to a disseminated pancreatic cancer, the more likely the cancerrelated complications (including new metastasis, intestinal obstruction, severe cachexia) might emerge in the clinical setting. Accordingly, systemic inflammation was previously suggested to be associated with tumor progression, metastasis along with resistance to chemotherapeutic agents largely through certain mechanisms including induction of epithelial-mesenchymal transition (characterized by changes in intercellular adhesion molecules including switch from E-cadherin to N-cadherin potentially allowing tumoral invasiveness and metastasis) along with stimulation of certain specific cells including cancer associated fibroblats (CAF) (promoting tumoral growth factors) and tumor associated macrophages (TAM) (primarily located around tumor margins and blood vessels, and facilitate metastasis through degradation of extracellular matrix and basement membrane) [60].

Lastly, an existing systemic inflammation potentially denotes myocardial tissue frailty (in correlation with the severity of 
myocardial inflammation) and substantial wall stress (particularly in those with extensive myocardial involvement or intraventricular gradient) [36-38], and hence; might portend a higher risk for deadly mechanical complications including free wall rupture. General mechanisms of inflammation-related adverse events in the setting of TTS are summarized in Figure 3.

\section{Potential therapeutic implications: Vasodilatory and anti-inflammatory strategies}

As expected, enhanced systemic inflammation in patients with TTS might potentially have therapeutic implications. In general, eradication of the inflammatory source (infection, malignancy), if possible, should be the primary goal in this setting to improve the overall prognosis. In particular, potential association of systemic inflammation with CSF pattern might particularly indicate certain vasodilator strategies in TTS patients with substantial levels of inflammation markers and/or serum catecholamine levels (or associated mediators including neuropeptide-Y) in an effort to improve short-term prognosis in these patients $[4,56,57,59]$.

In the absence of an overt inflammatory source, anti-inflammatory agents including, colchicine, steroids, certain cytokine blockers or modulators (canakinumab (IL-1 $\beta$ monoclonal antibody), tocilizumab (IL-6 receptor blocker)), that were all previously demonstrated to have favorable therapeutic effects in other cardiovascular inflammatory conditions [1,61], might also improve the overall prognosis of TTS with severe systemic inflammation. Since patients with TTS might suffer persistent systemic inflammation (and subclinical myocardial dysfunction) associated with long-term adverse events and poor quality of life [2,9,29], therapeutic benefits of these anti-inflammatory strategies [8] and conventional agents (including $\beta$ blockers and renin-angiotensin system (RAS) blockers) might potentially extend beyond the acute disease course suggesting their long-term use in clinical practice [2]. On the other hand, the impact of conventional agents on TTS recurrence currently remains to be fully established [20]. Taken together, therapeutic benefits of anti-inflammatory strategies currently remain speculative, and should be tested through clinical studies.

\section{Conclusions}

To date, systemic inflammation has been an underrated phenomenon in TTS as opposed to other cardiovascular conditions including ACSs and HF. However, growing body of evidence also suggests particular relevance of systemic inflammation in patients with TTS both in the short and long term. Clinically, there potentially exists multiple sources of systemic inflammation (mostly in combination) usually serving as contributors to and/or consequences of TTS evolution. Among these sources, myocardial inflammation has emerged as a novel phenomenon with important histopathological and clinical implications, and might be regarded as an obscure mechanism of systemic inflammation in the setting of TTS. In general, systemic inflammation might have important mechanistic, prognostic as well as therapeutic implications in

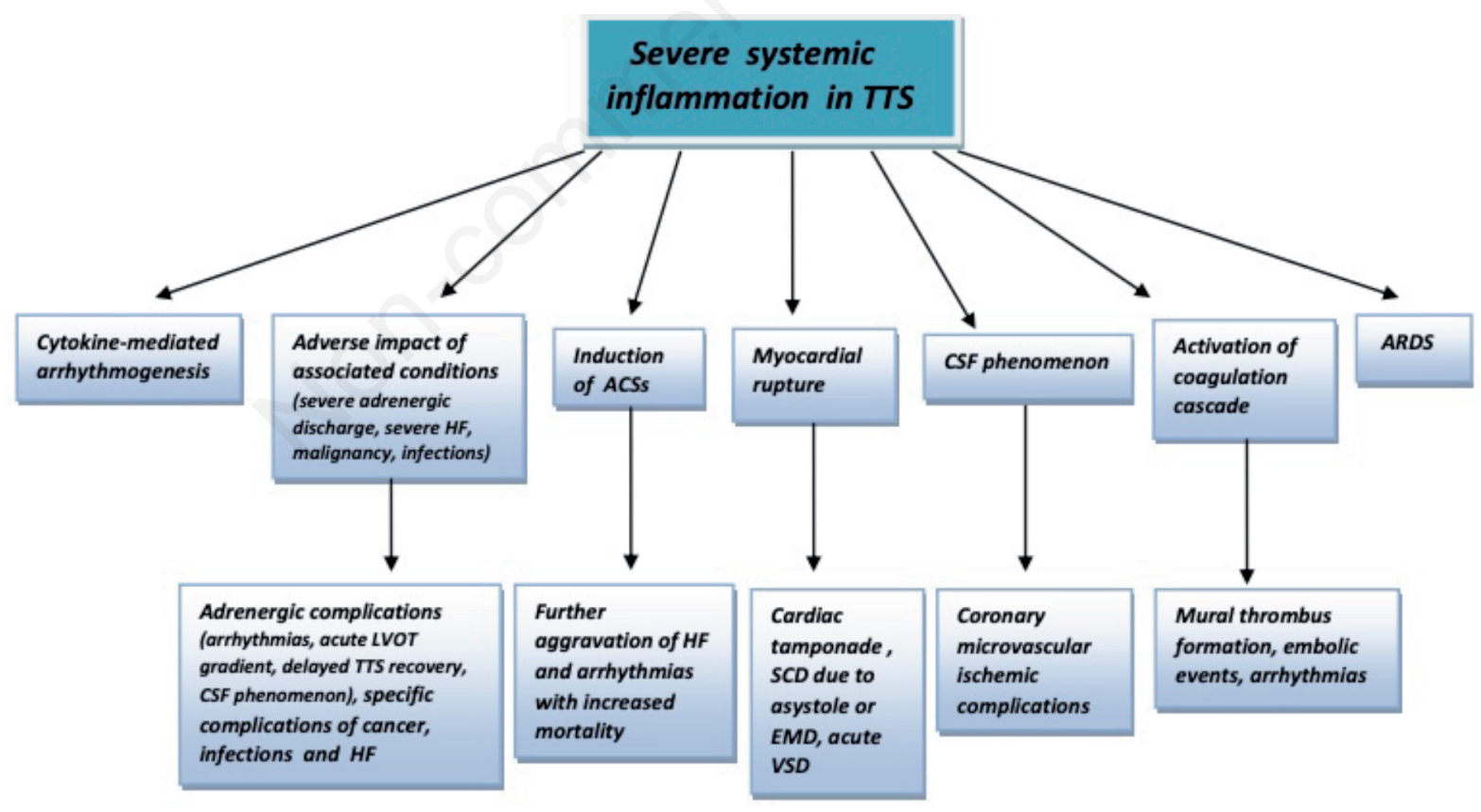

ARDS; Acute respiratory distress syndrome, TTS; takotsubo syndrome, ACS; acute coronary syndrome,

CSF; coronary slow flow, LVOT; left ventricular outflow tract, VSD; ventricular septal defect

HF; heart failure, SCD; sudden cardiac death, EMD; electromechanical dissociation,

Figure 3. Fundamental mechanisms of inflammation-related adverse events in the setting of takotsubo syndrome (TTS). 
patients with TTS. However, future studies are still needed to establish further aspects of systemic inflammation and its implications in these patients.

\section{References}

1. Yalta T, Yalta K. Systemic inflammation and arrhythmogenesis: a review of mechanistic and clinical perspectives. Angiology 2018;69:288-96.

2. Yalta K, Yilmaztepe M, Zorkun C. Left ventricular dysfunction in the setting of takotsubo cardiomyopathy: a review of clinical patterns and practical implications. Card Fail Rev 2018;4:14-20.

3. Santoro F, Guastafierro F, Zimotti T, et al. Neutrophil/lymphocyte ratio predicts in-hospital complications in Takotsubo syndrome. Results from a prospective multi-centerregistry. Clin Cardiol 2020;10.1002/clc.23442.

4. Yalta K, Yalta T. Physically triggered takotsubo cardiomyopathy has a worse prognosis: Potential roles of systemic inflammation and coronary slow flow phenomenon. Int J Cardiol 2017;242: 31-32.

5. Santoro F, Tarantino N, Ferraretti A, et al. Serum interleukin 6 and 10 levels in Takotsubo cardiomyopathy: Increased admission levels may predict adverse events at follow-up. Atherosclerosis 2016;254:28-34.

6. Yalta K, Yalta T. Cancer and takotsubo cardiomyopathy: More questions than answers. Int J Cardiol 2017;242:13.

7. Sattler K, El-Battrawy I, Lang S, et al. Prevalence of cancer in Takotsubo cardiomyopathy: Short and long-term outcome. Int J Cardiol 2017;238:159-65.

8. Scally C, Abbas H, Ahearn T, et al. Myocardial and systemic inflammation in acute stress-induced (Takotsubo) cardiomyopathy. Circulation 2019;139:1581-92.

9. Fernández-Ruiz I. Inflammation linked to Takotsubo. Nat Rev Cardiol 2019;16:5.

10. Kim MH, Gorouhi F, Ramirez S, et al. Catecholamine stress alters neutrophil trafficking and impairs wound healing by $\beta 2-$ adrenergic receptor-mediated upregulation of IL-6. J Invest Dermatol 2014;134:809-17.

11. Flierl MA, Rittirsch D, Nadeau BA, et al. Phagocyte-derived catecholamines enhance acute inflammatory injury. Nature 2007; 449:721-5.

12. Bergmann M, Sautner T. Immunomodulatory effects of vasoactive catecholamines. Wien Klin Wochenschr 2002;114:752-61.

13. $\mathrm{Ng} \mathrm{TM}$, Toews ML. Impaired norepinephrine regulation of monocyte inflammatory cytokine balance in heart failure. World J Cardiol 2016;8:584-9.

14. von Haehling S, Genth-Zotz S, Bolger AP, et al. Effect of noradrenaline and isoproterenol on lipopolysaccharide-induced tumor necrosis factor-alpha production in whole blood from patients with chronic heart failure and the role of beta-adrenergic receptors. Am J Cardiol 2005;95:885-9.

15. Nakano $T$, Onoue $K$, Nakada $Y$, et al. Alteration of $\beta$ Adrenoceptor Signaling in Left Ventricle of Acute Phase Takotsubo Syndrome: a Human Study. Sci Rep 2018;8:12731.

16. Pongratz G, Straub RH. The sympathetic nervous response in inflammation. Arthritis Res Ther 2014;16:504.

17. Sung CP, Arleth AJ, Feuerstein GZ. Neuropeptide Y upregulates the adhesiveness of human endothelial cells for leukocytes. Circ Res 1991;68:314-8.

18. Burgdorf C, Kurowski V, Bonnemeier H, et al. Long-term prognosis of the transient left ventricular dysfunction syn- drome (Tako-Tsubo cardiomyopathy): focus on malignancies. Eur J Heart Fail 2008;10:1015-9.

19. Romero-Bermejo FJ, Ruiz-Bailen M, Gil-Cebrian J, et al. Sepsisinduced cardiomyopathy. Curr Cardiol Rev 2011;7:163-83.

20. Ciutac AM, Dawson D. The role of inflammation in stress cardiomyopathy. Trends Cardiovasc Med 2020;S1050-1738(20) 30042-6.

21. Geng S, Mullany D, Fraser JF. Takotsubo cardiomyopathy associated with sepsis due to Streptococcus pneumoniae pneumonia. Crit Care Resusc 2008;10:231-4.

22. Yalta K, Gürdoğan M. Plasma histone H4 and 1ts 1mplications in the setting of sepsis-related myocardial dysfunction. Balkan Med J. 2020;37:119-20.

23. Li Y, Ge S, Peng Y, Chen X. Inflammation and cardiac dysfunction during sepsis, muscular dystrophy, and myocarditis. Burns Trauma 2013;1:109-21.

24. Faqihi F, Alharthy A, Alshaya R, et al. Reverse takotsubo cardiomyopathy in fulminant COVID-19 associated with cytokine release syndrome and resolution following therapeutic plasma exchange: a case-report. BMC Cardiovasc Disord 2020;20:389.

25. Yalta K, Yetkin E, Taylan G, et al. Acute autoimmune reaction: An obscure mechanism of COVID-19 related myocardial injury. Heart eLetters. Available from: https://heart.bmj.com/content/ 106/15/1154.responses\#acute-autoimmune-reaction-anobscure-mechanism-of-covid-19-related-myocardial-injury-

26. Desai HD, Jadeja DM, Sharma K. Takotsubo syndrome a rare entity in patients with COVID-19: An updated review of casereports and case-series. Int J Cardiol Heart Vasc 2020;29: 100604

27. Wilson HM, Cheyne L, Brown PAJ, et al. Characterization of the myocardial inflammatory response in acute stress-induced (takotsubo) cardiomyopathy. JACC Basic Transl Sci 2018;3:766-78.

28. Scally C, Ahearn T, Rudd A, et al. Right ventricular involvement and recovery after acute stress-1nduced (tako-tsubo) cardiomyopathy. Am J Cardiol 2016;117:775-80.

29. Neil CJ, Nguyen TH, Singh K, et al. Relation of delayed recovery of myocardial function after takotsubo cardiomyopathy to subsequent quality of life. Am J Cardiol 2015;115:1085-9.

30. Eitel I, Lücke C, Grothoff M, et al. Inflammation in takotsubo cardiomyopathy: insights from cardiovascular magnetic resonance imaging. Eur Radiol 2010;20:422-31.

31. Eitel I, von Knobelsdorff-Brenkenhoff F, Bernhardt P, et al. Clinical characteristics and cardiovascular magnetic resonance findings in stress (takotsubo) cardiomyopathy. JAMA 2011;306:277-86.

32. Nakamori S, Matsuoka K, Onishi K, et al. Prevalence and signal characteristics of late gadolinium enhancement on contrastenhanced magnetic resonance imaging in patients with takotsubo cardiomyopathy. Circ J 2012;76:914-21.

33. Foreman RD, Garrett KM, Blair RW. Mechanisms of cardiac pain. Compr Physiol 2015;5:929-60.

34. Pessoa PM, Xavier SS, Lima SL, et al. Assessment of takotsubo (ampulla) cardiomyopathy using iodine-123 metaiodobenzylguanidine scintigraphy. Acta Radiol 2006;47:1029-35.

35. Manabe O, Naya M, Oyama-Manabe N, et al. The role of multimodality imaging in takotsubo cardiomyopathy. J Nucl Cardiol 2019;26:1602-16.

36. Murakami T, Yoshikawa T, Maekawa Y, et al. Characterization of predictors of in-hospital cardiac complications of takotsubo cardiomyopathy: multi-center registry from Tokyo CCU Network. J Cardiol 2014;63:269-73. 
37. Van Linthout S, Tschöpe C. Inflammation - Cause or consequence of heart failure or both? Curr Heart Fail Rep 2017;14: 251-65.

38. Yoshida T, Friehs I, Mummidi S, et al. Pressure overload induces IL-18 and IL-18R expression, but markedly suppresses IL-18BP expression in a rabbit model. IL-18 potentiates TNF- $\alpha$-induced cardiomyocyte death. J Mol Cell Cardiol 2014;75:141-51.

39. Yalta K, Yetkin E, Yalta T. Recurrent takotsubo cardiomyopathy: Further insights into morphological patterns. Cardiovasc Pathol 2020;48:107225.

40. Yalta K, Gurdogan M, Palabiyik O. Apical aneurysm or transient apical ballooning? Potential dilemma in risk stratification of hypertrophic cardiomyopathy. Heart 2020;106:1111.

41. Yalta K, Ucar F, Yilmaztepe M, et al. Takotsubo cardiomyopathy and acute coronary syndromes: Are they always mutually exclusive? Indian Heart J 2018;70:326-27.

42. Yalta K, Yilmaztepe M, Ucar F, et al. Tako tsubo cardiomyopathy? Acute myocarditis? Or both? Not so easy to diagnose in certain settings. Int J Cardiovasc Res 2017;6:3.

43. Chhabra L. Myopericarditis and Takotsubo cardiomyopathy association. Int J Cardiol 2015;186:143.

44. de Miguel V, Arias A, Paissan A, et al. Catecholamine-induced myocarditis in pheochromocytoma. Circulation 2014;129: 1348-9.

45. Lee SH, Chen YC, Chen YJ, et al. Tumor necrosis factor-alpha alters calcium handling and increases arrhythmogenesis of pulmonary vein cardiomyocytes. Life Sci 2007;80:1806-15.

46. Grandy SA, Brouillette J, Fiset C. Reduction of ventricular sodium current in a mouse model of HIV. J Cardiovasc Electrophysiol 2010;21:916-22.

47. Acar GR, Akkoyun M, Nacar AB, et al. Evaluation of Tp-e interval and Tp-e/QT ratio in patients with rheumatoid arthritis. Turk Kardiyol Dern Ars 2014;42:29-34.

48. Panoulas VF, Toms TE, Douglas KM, et al. Prolonged QTc interval predicts all-cause mortality in patients with rheumatoid arthritis: an association driven by high inflammatory burden. Rheumatology (Oxford) 2014;53:131-7.

49. Okazaki R, Iwasaki YK, Miyauchi Y, et al. Lipopolysaccharide induces atrial arrhythmogenesis via down-regulation of L-type Ca2+ channel genes in rats. Int Heart J 2009;50:353-63.

50. Lew WY, Yasuda S, Yuan T, et al. Endotoxin-induced cardiac depression is associated with decreased cardiac dihydropyridine receptors in rabbits. J Mol Cell Cardiol 1996;28:1367-71.

51. Foley ED, Diaz R, Castresana MR. Prolonged circulatory support with an Impella assist device in the management of cardiogenic shock associated with takotsubo syndrome, severe sepsis and acute respiratory distress syndrome. SAGE Open Med Case Rep 2017;5:2050313X17741013.

52. Nakamura M, Nakagaito M, Hori M, et al. A case of Takotsubo cardiomyopathy with cardiogenic shock after influenza infection successfully recovered by IMPELLA support. J Artif Organs 2019;22:330-3.

53. van den Oever IA, Sattar N, Nurmohamed MT. Thromboembolic and cardiovascular risk in rheumatoid arthritis: role of the haemostatic system. Ann Rheum Dis 2014;73:954-57

54. Fitzgibbons TP, Edwards YJK, Shaw P, et al. Activation of inflammatory and pro-thrombotic pathways in acute stress cardiomyopathy. Front Cardiovasc Med 2017;4:49.

55. Yalta K, Yalta T, Gurdogan M. Takotsubo syndrome coexıstıng with acute myocardial infarction: an overlooked phenomenon in clinical practice. N Z Med J 2020;133:81-2.

56. Yalta K, Yilmaztepe M, Ucar F, et al. Coronaryslowflow in thesetting of Tako-tsubocardiomyopathy: A causativefactor? An innocentbystander? Or a prognosticsign? Int J Cardiol 2015; 198:229-31.

57. Yalta K, Sivri N, Yalta T. Neuropeptide Y-induced coronary microvascular dysfunction: a significant contributor to the adverse outcomes in stress cardiomyopathy? Int J Cardiol 2011;147:284

58. Kawaji T, Shiomi H, Morimoto T, et al. Clinical impact of left ventricular outflow tract obstruction in takotsubo cardiomyopathy. Circ J 2015;79:839-46.

59. Yalta K, Yalta T. Takotsubo cardiomyopathy and its implications in the setting of acute manic attack. Proc (Bayl Univ Med Cent) 2020;33:473-4.

60. Liu J, Lin PC, Zhou BP. Inflammation fuels tumor progress and metastasis. Curr Pharm Des 2015;21:3032-40.

61. Yalta K, Yilmaz MB, Yalta T, et al. Late versus early myocardial remodeling after acute myocardial infarction: a comparative review on mechanistic insights and clinical implications. J Cardiovasc Pharmacol Ther 2020;25:15-26. 